ÚLTIMA DÉCADA, N47, DICIEMBRE 2017, PP. 83-117

\title{
RELACIONES CONFLICTIVAS ENTRE JÓVENES EN SITUACIÓN DE POBREZA Y POLICÍA: SU ABORDAJE EN PROGRAMAS SOCIALES ${ }^{1}$
}

MARINA MEDAN ${ }^{2}$

\begin{abstract}
RESUMEN
Desde comienzos de 2000 se implementan en Argentina programas de inclusión y prevención social del delito destinados a que jóvenes en "riesgo" gesten un "proyecto de vida” alrededor de la inclusión educativa, laboral y la revisión de sus vínculos. Paralelamente, estudios sobre la sociabilidad juvenil en contextos de desigualdad evidencian que la conflictiva relación con la policía es un problema central de su cotidianeidad. Este artículo discute la limitada forma en que estos programas, que procuran proteger a los jóvenes, abordan dicha relación. Se analizan datos de investigaciones cualitativas realizadas en Buenos Aires entre 2007 y 2016.
\end{abstract}

PALABRAs ClaVe: JUVENTUd, PREVENCIÓN SOCIAL DEL DELITO, VIOLENCIA INSTITUCIONAL

\footnotetext{
1 Agradezco especialmente a Tomás Bover por los comentarios a este trabajo. 2 Dra. en Ciencias Sociales, Universidad de Buenos Aires. Investigadora del Consejo Nacional de Investigaciones Científicas y Técnicas (Argentina), en el CEDeSI de la Universidad Nacional de San Martín. Además, es docente de la Universidad de Buenos Aires. Correo electrónico: marinamedan@conicet.gov.ar
} 


\title{
RELAÇÕES CONFLITIVAS ENTRE JOVENS EM SITUAÇÕES DE POBREZA E A POLÍCIA: SUA ABORDAGEM EM \\ PROGRAMAS SOCIAIS
}

\begin{abstract}
RESUMO
Desde começos do ano 2000 foram implementados na Argentina programas de inclusão e prevenção social do crime destinados a que os jovens em "risco" gestem um "projeto de vida" ao redor da inclusão educativa, do trabalho e a revisão de seus vínculos. Paralelamente, estúdios sobre a sociabilidade juvenil em contextos de desigualdade evidenciam que a conflitiva relação com a polícia é um problema central de sua cotidianidade. Este artigo discute a limitada forma em que estes programas, que procuram proteger os jovens, abordam esta relação. São analisados dados de pesquisas qualitativas realizadas em Buenos Aires entre 2007 e 2016.
\end{abstract}

PALAVRAS-CHAVE: JUVENTUDE, PREVENÇÃO SOCIAL DO CRIME, VIOLÊNCIA

INSTITUCIONAL.

\section{CONFLICTIVE RELATIONSHIPS AMONG POOR YOUNG INDIVIDUALS AND THE POLICE: THEIR APPROACH FROM SOCIAL PROGRAMS}

\begin{abstract}
Social crime inclusion and prevention programs oriented to young people have been applied in Argentina since early 2000s, in order young people at "risk" to build a "project of life" around educational, work and affective inclusion. In parallel, studies on young sociability within inequality contexts show this conflictive relationship with the police to be a central problem in their daily lives. This article discusses on the limitations of these programs, intended to protect young people, at the moment of tackling such relationship, from the analysis of data from qualitative research between 2007 and 2016 carried out in Buenos Aires.
\end{abstract}

KEYWORDS: YOUTH, SOCIAL CRIME PREVENTION, INSTITUTIONAL VIOLENCE. 


\section{INTRODUCCIÓN}

Este artículo se inscribe en los debates sobre el gobierno de las poblaciones, especialmente de jóvenes en contextos de desigualdad y trata sobre la forma en que los programas de inclusión y prevención social del delito juvenil abordan la conflictiva relación que los jóvenes beneficiarios de dichos dispositivos mantienen con la policía.

Desde hace al menos 15 años, en contextos de desigualdad sostenida como en Argentina, las políticas sociales y de seguridad se imbrican para gobernar a las nuevas generaciones. Así resultan dispositivos estatales en los que se combinan objetivos de control y de gestión del delito con otros de bienestar, cuidado y restitución de derechos vulnerados. En este artículo me refiero concretamente a programas de prevención social del delito y de inclusión social juvenil que se implementan territorialmente en barrios populares del gran Buenos Aires. Éstos se centran en la idea de "proyecto de vida” y orientan sus acciones hacia la inclusión educativa y laboral de los adolescentes y jóvenes, procuran el acceso a la salud y el tratamiento de consumos problemáticos de sustancias, e insisten en la revisión de las relaciones vinculares de los jóvenes con sus familias, comunidades y pares que atentan contra ese "proyecto". Los programas procuran, a grandes rasgos, proteger a los jóvenes de los riesgos de su entorno y fortalecerlos para que puedan convertirse en adultos autónomos y responsables. Mientras en otros trabajos he descripto y analizado los modos en que los programas procuran proteger (y controlar) a los y las jóvenes de situaciones calificadas por los programas como “riesgosas” (la desafiliación institucional, el delito, la maternidad adolescente, entre otros), en este artículo me interesa indagar como los programas abordan una relación que se presenta como problemática para los jóvenes: la que mantienen con la policía. La hipótesis que guía la indagación es que la centralidad que dicha relación tiene para los jóvenes no tiene un correlato 
de igual magnitud en la intervención institucional.

\section{ANTECEDENTES}

Los estudios sociales en Argentina han prestado atención al delito juvenil desde hace al menos 15 años y lo han caracterizado con mucho detalle, sociológica y antropológicamente, en trabajos que ya son clásicos (Kessler, 2004, Míguez, 2010, entre otros). ${ }^{3}$ En ellos, la relación con la policía es uno de los aspectos centrales. Me interesa mostrar el despliegue de esta relación en, al menos, tres formas. En primer lugar, el contacto con la policía suele ser el inicio de la socialización legal de los jóvenes, especialmente varones, de sectores populares (Zabaleta y otros, 2016:211, Alvarado Mendoza, 2015) inclusive, de aquellos que no cometen delitos (Kessler, 2004). Respecto de los que sí, es la institución que determina su ingreso al sistema penal, ya que es el primer eslabón de la selección de lo criminalizante (Saín, 2015: 123, 127; Daroqui y otros, 2012). ${ }^{4}$ En segundo lugar, y en relación con el papel que la policía tiene en los procesos de socialización legal, los estudios muestran que además de por enfrentamientos, los jóvenes también tienen relaciones -no equipotenciales- de “cooperación” con la policía, en función de las actividades ilegales de ésta. Además, las relaciones con la policía son cercanas no sólo por la cotidianeidad de su contacto, sino por ciertas dinámicas de acción compartidas. No sólo porque, desde nuestra perspectiva, los jóvenes que tienen prácticas delictivas no

3 Ellos, por su parte, han retomado y rediscutido una tradición de larga data, amplia y diversa, de estudios sociales y criminológicos sobre la delincuencia juvenil, especialmente desarrollada en Estados Unidos y posteriormente en Europa, con diversas teorías más vinculadas al positivismo o el interaccionismo simbólico, entre otras.

4 Respecto a las dinámicas de criminalización que operan sobre los jóvenes, la policía es sin dudas un actor clave, aunque no el único. Desde esta perspectiva cabría recuperar los aportes de la teoría del etiquetamiento la cual indaga en torno a quiénes deciden qué tipo de comportamiento es desviado o eventualmente peligroso, y además considera cómo estas asignaciones de "etiquetas" inciden, a su vez, en el modo en que las personas etiquetadas se comportan (Lemert, 1961, Becker, 2014). 
forman parte de otra cultura distinta de la "convencional” (Matza, 2014) que regularía al resto de la población y las instituciones del Estado como la policía. Además, las relaciones con la policía son cercanas no sólo por la cotidianeidad de su contacto, sino por ciertas dinámicas de acción compartidas. No sólo porque, desde nuestra perspectiva, los jóvenes que tienen prácticas delictivas no forman parte de otra cultura distinta de la "convencional” (Matza, 2014) que regularía al resto de la población y las instituciones del Estado como la policía. Sino porque el desdibujamiento de la ley y de las fronteras morales entre lo legal y lo ilegal, lo legítimo y lo ilegítimo que incide en las acciones delictivas juveniles también es, para los jóvenes, pero como veremos más adelante para gran parte de la sociedad, una característica de la policía. Para los jóvenes, la policía es una institución que poco tiene que ver con la ley, y a la consideran, más vale, otra banda, más organizada y con mejores armas que la de ellos (Kessler, 2004). De la relación cotidiana y conflictiva con la policía, algunos jóvenes, al calor del avance de la visibilización de los derechos humanos, han aprendido sobre sus éstos y logran responder a la policía o al menos resistirse (Zabaleta y otros, 2016; Kessler y Dimarco, 2013) y hasta en casos excepcionales organizarse en contra de los abusos (Lerchundi y Bonvillani, 2015). En tercer lugar, cuando no se los criminaliza judicialmente, ni existe detención, ni delito, hay hostigamiento policial cotidiano (Kessler y Dimarco, 2013; Rodríguez Alzuelta, 2014), y la relación que se configura entre policías y jóvenes es una que los sobrevigila y subprotege (Cozzi y Mistura, 2014). La relación con la policía incluso tiene su capítulo en estudios generales sobre la cotidianeidad de los jóvenes (que no necesariamente se ocupan del delito o la seguridad), especialmente en aquellos que consideran el uso del espacio público y formas de circulación de jóvenes (Chaves, 2010; Segura, 2012; Di Leo y Camarotti, 2013). Por último, vale considerar especialmente los trabajos que, enmarcados en el campo de los derechos humanos, se ocupan de las diferentes formas de violencia institucional y dentro de ellas, la relación entre jóvenes y 
policías constituye la forma paradigmática (CELS, 2013). ${ }^{5} \mathrm{Al}$ respecto, vale destacar que mientras el discurso de derechos humanos ha ido permeando en la sociedad argentina en los últimos años, y la lucha contra la violencia institucional se ha formalizado, cuando la policía comete abusos en nombre de la prevención sobre ciertos sectores -y especialmente en nombre de la prevención del delito urbano-, esas prácticas no son condenadas socialmente (Kessler y Dimarco, 2013); es más, son amparadas en la impunidad porque se dirigen a las "clases peligrosas" (Saín, 2015:133). Esta legitimidad sobre la ilegalidad policial no puede responder simplemente a la autonomía policial, requiere un consenso social que no se puede soslayar.

La policía, por su parte, también ha sido objeto de numerosas investigaciones (desde la criminología, sociología y antropología). Por la particularidad de cada institución policial, y su configuración histórica, aquí resultan centrales los antecedentes argentinos que reconstituyen la configuración de nuestra policía en términos históricos y sociales y que vinculan su entidad, accionar y legitimidad con el entorno social en el cual se inscribe (Saín, 2015; Galvani, 2016; Sirimarco, 2010; Bover, 2016). En términos de misión, la policía en Argentina sigue una orientación más ligada a la garantía del orden del Estado que a la de los derechos del pueblo; respecto del tipo de delitos que persigue, por su estructura organizacional y funcional, prefiere o los delitos de poca monta, o aquellos contra la propiedad y contra la vida por sobre los del crimen organizado, priorizando el despliegue territorial sobre las actividades de inteligencia (Saín, 2015: 151, 169 y ss.). El foco en los

5 Aunque coincido en la dificultad de precisar una definición de la violencia por fuera de su referencia empírica (Garriga Zucal y Noel, 2010, Perelman y Tufró, 2016), tomo los aportes que se refieren a ella como una acción relativa a lo ilegítimo, con carácter paradójico en la medida en que construye y destruye el lazo social, y como una agresión que un destinatario preferiría no sufrir. Específicamente respecto de la violencia institucional, se la caracteriza por la "existencia de una práctica específica (asesinato, aislamiento, tortura, etc.), la participación de funcionarios públicos (que llevan adelante o prestan aquiescencia) y el contexto de restricción de autonomía y libertad (situaciones de detención, de internación, de instrucción, etc.)” (Perelman y Tufró, 2016). 
delitos callejeros y contra la vida, también coincide con el clamor popular, dado que son los que más influyen en la sensación de inseguridad (Kessler, 2009). ${ }^{6}$ Por el tipo de misión y funcionamiento de la policía, los jóvenes resultan su principal grupo de atención en la medida en que son los que están involucrados en los delitos que se persiguen y que logran conjurarse. Los protagonistas del delito amateur (Kessler, 2004) son jóvenes, y son quienes con prácticas toscas, improvisadas y en el ámbito callejero son los más fáciles de captar por la policía (Saín, 2015: 126). Esto se suma a que, históricamente, ha sido necesaria la estigmatización de ciertos sectores y la creación de “enemigos” -no sólo de parte la policía - que permitan la actuación legítima de la policía; mientras otrora fueron los vagos, luego los militantes anarquistas, y luego los terroristas, actualmente son los jóvenes delincuentes (Saín, 2015; Galvani, 2016: 112).

Ahora bien, en los trabajos mencionados, el escenario de la interacción que encuentra a la policía y a los jóvenes, no aparece conformado por ningún otro actor estatal más allá del policial. De allí que una de las lecturas que se extiende con frecuencia es que el Estado se relaciona con estos jóvenes solamente mediante su fuerza represiva (Auyero y Berti, 2013). En otros trabajos hemos discutido la invisibilización que en dicho tipo de estudios hay de las estrategias estatales que se despliegan en los territorios empobrecidos y que procuran la protección de los jóvenes (Llobet y Medan, 2015; Medan, 2014). Sin ir más lejos, sostenemos que estos programas de inclusión social y prevención social del delito que se implementan territorialmente en los barrios populares son una forma de hacerle frente a la versión más dura del Estado. Ahora bien, lo que también notamos, y sobre lo que quiero discutir aquí es que el cuidado que propician frente a los "riesgos” que aquejan a los jóvenes no

6 No obstante la coincidencia entre demandas sociales y acciones policiales, la sociedad no tiene una buena percepción de la policía, aunque paradójicamente crea que la policía es quien tiene que garantizar la seguridad y tienda a demandar su intervención en casi cualquier situación conflictiva (Saín, 2015:39). A su vez, la policía es consciente de estas percepciones negativas y las siente profundamente injustas (Galvani, 2016). 
parece trascender aquellos relacionados con la exclusión educativa, laboral, o el sostenimiento de vínculos “problemáticos”.

Así las cosas, a pesar de la evidencia que la literatura nos ofrece sobre las relaciones entre jóvenes y policía, los estudios -propios y ajenos- sobre la regulación estatal de jóvenes a partir de programas sociales y de prevención del delito (Mancini, 2015; Ayos, 2010; Llobet, 2013; Medan, 2014) no han avanzado suficientemente en caracterizar cómo estas relaciones -que configuran de algún modo el "riesgo” en el que los y las jóvenes están insertos- aparecen en dichos programas y son abordadas. Este artículo procura contribuir en este sentido y, en términos más amplios, a la caracterización de los vínculos entre los sistemas sociales y penales o de securitarios que se ocupan de la gestión de las juventudes en situación de pobreza.

\section{HIPÓTESIS Y PERSPECTIVA TEÓRICA}

La hipótesis de trabajo que guía estas reflexiones (que no podrá ser verificada o refutada en estas líneas, pero sí comenzada a pesquisar) es que el modo en que la policía es considerada por los programas sociales destinados a jóvenes, incide desfavorablemente en las capacidades de los dispositivos de inclusión de alcanzar los objetivos institucionales. La indagación específica sobre la relación con la policía, se inscribe en un plan de investigación más amplio sobre cómo se articulan estrategias estatales, que combinan objetivos de inclusión social y prevención del delito, con otros tipos de intervenciones estatales, y redes de relaciones informales, ilegales y comunitarias. En general, procura reconocer los distintos proyectos de gobierno $^{7}$ que existen en la

7 Aquí se entiende al término gobierno en el sentido amplio foucaultiano, como la forma que estructura el campo posible de acción, que guía la conducta de uno mismo y/o sobre otras personas (Foucault, 2003: 141). Con "proyectos de gobierno" me refiero a actores -encarnados en personas, figuras o instituciones-, a redes de relaciones que incluyen formas de jerarquía, sujeción, afinidad, empatía, y a universos de sentidos normativos, morales y sensibles. 
comunidad (Rose, 1996; de Marinis, 1999) para los y las jóvenes y asume que mientras los programas de inclusión social y prevención social del delito buscan que a través de su intervención los/as jóvenes armen un "proyecto de vida alternativo al delito”, una pluralidad de regulaciones (Das y Poole, 2008) parecen estar disputándoles la clientela (Haney, 2010), y ofreciendo a los/as jóvenes otras formas de sociabilidad que obstaculizarían el "éxito” de las intervenciones. Uno de los objetivos del proyecto general supone identificar y describir esas “instancias de regulación”, y el modo en que estos programas lidian con ellas. En este sentido, este trabajo sobre el modo de percibir y abordar la relación de los jóvenes con la policía, colabora con él.

En la medida en que este artículo trata con formas de intervención estatales que pueden estar orientadas por principios funcionales e ideológicos muy disímiles -para decirlo esquemáticamente, el ala blanda y el ala dura del Estado-, considero fundamental utilizar una definición compleja de Estado. Para ello me nutro de un marco interpretativo deudor de los aportes de la teoría feminista al estudio del gobierno y el Estado que lo entienden como un ente multidimensional, dispuesto en capas y compuesto de aparatos que actúan de forma no siempre coherente; además, estos estudios señalan no sólo los efectos redistributivos de las políticas sociales y penales sino sus patrones de reconocimiento y de representación (Haney 2004, Fraser, 1991; Llobet, 2013). De allí que estos aportes informen el estudio sobre cómo los dispositivos estatales interpretan necesidades, construyen sujetos de regulación y redistribuyen recursos materiales y simbólicos. Por otro lado, atiendo a los paralelismos que es posible encontrar entre los sistemas de bienestar y punitivos (Haney, 2004), y a su capacidad de ejercer el control de las poblaciones por medio de la contradicción y ambigüedad. Haney explica la efectividad, para el gobierno, que supone el hecho de que los sujetos sean interpelados sobre un mismo fenómeno por distintas agencias con distintas expectativas (por ejemplo, por un lado la que tienen los sistemas sociales sobre la capacidad de los jóvenes de lidiar con el riesgo de su entorno, y las que tiene la policía o el sistema socio 
penal, en general).

\section{MATERIALES Y ENFOQUE METODOLÓGICO.}

Los datos provienen de investigaciones ${ }^{8}$ cualitativas propias, realizadas desde 2007 y aún vigentes, sobre los modos de regulación de las juventudes de sectores populares mediante programas sociales y de prevención del delito, implementados territorialmente en Avellaneda, Morón y San Martín en provincia de Buenos Aires, Argentina. Las investigaciones de las que surgen los datos supusieron un diseño del tipo de caso instrumental y múltiple (Stake, 1998); al mismo tiempo, dado que las hipótesis de trabajo suponían que ciertas claves de comprensión del caso estaban fuera de él y debían buscarse en el “exterior”, los estudios tomaron aspectos de los diseños propios del estudio de caso extendido (Burawoy, 1998). Las técnicas de construcción de datos fueron: a) observación participante (OP) en el campo, directa, no controlada, no estructurada, no mediada y del tipo participante como observador. Los datos construidos en la OP se volcaron en registros de campo (RC). Las notas

8 Las investigaciones propias fueron/son: entre 2008 y 2011 “ ¿Transferencia de ingresos para prevenir el delito? Participación juvenil, trabajo y masculinidades en el Programa Comunidades Vulnerables” (beca doctoral tipo I CONICET); entre 2011 y 2013 "Prevención del delito con transferencias condicionadas de ingresos: negociaciones entre un programa social y jóvenes de sectores populares" (beca doctoral tipo II CONICET); 2014-2015 “¿Quién gobierna a la "juventud en riesgo”? Programas estatales y redes comunitarias, informales e ilegales compitiendo por las fidelidades de hombres y mujeres jóvenes en territorios empobrecidos” (beca postdoctoral CONICET); 2015-2018 "La regulación social de las y los jóvenes en condiciones de desigualdad. Articulaciones inestables entre políticas de "inclusión” para prevenir el delito juvenil y otras prácticas estatales y formas de sociabilidad cercana” (PICT 0739-2015) Dirección a mi cargo y equipo V. Llobet, C. Gaitán, y B. Focas. La investigaciones colectivas fueron entre 2010-2012 "La participación de las/os adolescentes en los programas de inclusión social y las identidades de género" (PIP 2010-2012 N 11220090100520, dirección V. Llobet); entre 2011 y 2016 "La protección de derechos de niños, niñas y adolescentes y las interpretaciones del "bienestar de la infancia": disputas e interacciones en la construcción de los Sistemas locales de protección de derechos” (PICT 2281-2011, dirección V. Llobet). 
observacionales, teóricas y metodológicas se clarificaron a través de entrevistas en profundidad, semi-estructuradas y abiertas para lograr entre un medio y alto grado de espontaneidad a funcionarios/as (directores de área) y operadores (profesionales y no profesionales pero que trabajan directamente en los barrios en donde residen los jóvenes destinatarios) de áreas sociales de infancia y adolescencia, de nivel municipal y provincial, y de áreas nacionales de justicia y seguridad, y a jóvenes beneficiarios/as. Las entrevistas procuraron reponer el punto de vista de los actores, interpretando sus testimonios como una construcción social multideterminada. Estos materiales se asumieron no como meros modos de nombrar la vida social sino como fuerzas activas que le dan forma (Fraser y Gordon, 1994) y que suponen construcción de hegemonía.

El trabajo en Avellaneda se realizó en dos etapas, entre 2007 y $2009^{9}$ y en 2014, ${ }^{10}$ principalmente en un barrio y en menor medida en otro en donde se implementaba el mismo modelo de programa de prevención social del delito

9 Todos los RC de esa etapa están colocados sin fecha.

10 Los RC de 2014 están consignados con fecha. 
“Comunidades Vulnerables” (PCV). ${ }^{11}$ De la primera etapa surgieron 75 RC y 12 entrevistas en profundidad. Y en la segunda, 11 RC y 4 entrevistas. El trabajo de campo en Morón se realizó entre 2012 y 2014, y las referencias son respecto del Programa de Responsabilidad Social Compartida Envión, ${ }^{12}$ y del funcionamiento del Servicio Local ${ }^{13}$ del barrio en el que se hizo el estudio. Se analizaron 10 entrevistas a funcionarios y operadores y 4 RC de actividades del programa. En San Martín, el trabajo de campo ha sido continuo desde 2014,

11 El PCV fue creado en Argentina el 2001 por el Ministerio de Justicia y Derechos Humanos dentro del Plan Nacional de Prevención del delito (PNPD). Según su diseño (Dirección Nacional de Política Criminal, 2007) procuraba “promover la construcción de proyectos de vida que no impliquen prácticas violentas y delictivas y apoyar la búsqueda de formas de sustento económico por fuera de la actividad delictiva”. Sus destinatarios eran personas de entre 16 y 30 años, definidos como "adolescentes y jóvenes en situación de vulnerabilidad social en conflicto con la ley o en riesgo de estarlo”. La implementación que estudié consistía en encuentros grupales de dos horas, una o dos veces a la semana en distintas locaciones dentro de los barrios donde vivían los/as beneficiarios, y donde abordaban temáticas en torno a los ejes: trabajo, socio comunitario, vincular y judicial. Además, se sucedían charlas individuales con los/as jóvenes, relacionadas con el consumo y tratamiento de adicciones, o resolución y seguimiento de conflictos judiciales. Los/as jóvenes debían asumir un compromiso personal que podía tomar la forma de reinserción escolar, o laboral o realizar algún curso de formación profesional, o tratamiento de recuperación de adicciones o tareas comunitarias. El Programa ofrecía una transferencia condicionada de ingresos (TCI). El PCV funcionó en 6 barrios de la Ciudad de Buenos Aires, en 12 localidades bonaerenses, y en 4 ciudades del interior del país; alcanzó a unos 6000 beneficiarios, involucró a 70 personas en tareas de coordinación e implementación y nunca tuvo presupuesto propio. Desde 2008, cuando se desmanteló el programa a nivel nacional, pasó a dependencias municipales, y continúa funcionando -con otros nombres- en varios municipios argentinos, incluido aquel en donde se hizo el trabajo de campo.

12 Está dirigido a personas de 12 a 21 años en situación de vulnerabilidad social de la Provincia de Buenos Aires. Los objetivos oficiales del programa son: integrar a las/os jóvenes al sistema educativo, enseñarles un oficio y procurarles un espacio de contención donde puedan realizar actividades deportivas, recreativas y culturales. Depende del Ministerio de Desarrollo Social de Buenos Aires, pero es gestionado municipalmente. Comenzó a implementarse en 2009 y para fines de año 2012, se encontraba en 131 de los 134 distritos de dicha provincia. Es uno de los programas dirigidos a la población infanto-juvenil con mayor alcance y asignación presupuestaria del país, y brinda transferencias monetarias que son percibidas directamente por los destinatarios, como incentivo a la participación en el mismo y a condición de cumplir con las actividades pautadas.

13 Es un tipo de dispositivo territorial de dependencia municipal encargado de atender la vulneración de derechos de niños, niñas y adolescentes, creado en el marco del Sistema de protección y promoción de derechos de niñas, niños y adolescentes (Ley de la Provincia de Buenos Aires $n^{\circ}$ 13298). 
sobre diferentes programas y dispositivos. Para esta ponencia se consideraron especialmente 4 entrevistas realizadas al equipo de implementación de un Centro Juvenil ${ }^{14}$ en un barrio durante 2015, y los RC de las reuniones mensuales del Consejo Local de Derechos de Niñas, Niños y Adolescentes, ${ }^{15}$ y las reuniones de su comisión de Adolescencia (de frecuencia no periódica) durante 2014, 2015 y 2016.

La perspectiva teórico metodológica adopta aportes de la teoría fundamentada (Corbi y Strauss, 1990). Se procedió a identificar en los materiales construidos aquellos temas fundamentales en torno a las formas en que la cuestión de lo policial y la policía aparecía en las interacciones cotidianas en el seno de los programas. A partir de dicho análisis se identificaron tres grupos de datos: los modos en que la policía tomaba lugar en la vida cotidiana de los jóvenes a partir de los que ellos dejaban entrever en las dinámicas institucionales; las valoraciones de los agentes o profesionales de los programas sobre la policía y la relación con sus intervenciones sociales; y las formas en que institucionalmente se abordaba la relación entre jóvenes y policía. Dentro de este último grupo de datos, se identificaron tres sub categorías relativas a los modos de acción de parte de los programas.

14 Los Centros Juveniles de San Martín son dispositivos municipales de inclusión social dependientes de la Dirección de Políticas de Juventud que atienden diariamente a población socialmente vulnerable de entre 14 y 21 años, a quien le brindan asistencia alimentaria, y talleres recreativos y de formación, además de, en algunos casos, una transferencia condicionada de ingresos como apoyo económico y contrapartida a la participación. Los Centros Juveniles fueron creados en el marco de la implementación municipal del Programa provincial Envión (detallado en la nota 12).

15 Estos Consejos son parte del Sistema de Promoción y Protección Integral de derechos de la provincia de Buenos Aires, contemplado en los artículos 14 y 15 de la Ley Provincial 13.298 del 2005. Constituyen espacios de participación, veeduría y recomendación de políticas, que funcionan a nivel municipal en los que actores representantes de áreas estatales y de la sociedad civil se reúnen con una cierta periodicidad a discutir los problemas de la infancia y adolescencia. 


\section{Resultados: DE PRESENCIAS POLICIALES COTIDIANAS Y VALORACIONES Y ACCIONES INSTITUCIONALES}

En esta sección evidencio que en la vida cotidiana de los jóvenes que participan en estos programas la policía es un actor importante. En segundo lugar, presento las valoraciones y percepciones que los agentes que implementan los programas tienen sobre la policía, y las evaluaciones que hacen acerca de la factibilidad de mediar en las relaciones con los jóvenes. Finalmente muestro las formas en las que los programas abordan la relación entre jóvenes y policía, y sus variedades internas.

5.1 La relación con la policía como parte de la vida cotidiana de los jóvenes.

El contacto de los jóvenes con la policía es diario, y los datos analizados permiten agrupar estos contactos en: a) experiencias directas de interacción, que a su vez se han sedimentado de tal forma que se traducen en b) temores permanentes, y c) en experiencias indirectas, en la medida en que se refieren al contacto entre personas de su grupo familiar o cercano y la policía en las que ellos tienen alguna acción, o d) a partir de las cuales han tenido una experiencia traumática.

Respecto a los contactos directos, son detenidos por cartonear (R15), o “por portación de cara” (R28), o en reiteradas veces por averiguación de antecedentes (entrevista a $\mathrm{EA}^{16}$ ), o “por cualquier cosa” (R39), o por confusiones con otros jóvenes (entrevista a $\mathrm{HZ}$ ). Las detenciones son habituales, independientemente de lo que hagan o lo que no hagan, como

16 Se señalan las iniciales de los nombres y apellidos de los entrevistados para proteger su identidad. 
hostigamiento permanente, según una de las operadoras barriales del programa Comunidades Vulnerables. De allí que el temor sea uno de los sentimientos más frecuente que tienen respecto de la policía. Los jóvenes temen ser detenidos cuando van a la avenida principal a cuidar autos, o cuando les llegan citaciones a las casas que no entienden y prefieren esconderse por miedo a que la citación en realidad implique una detención (R27, R50). ${ }^{17}$ También sucede que se alejan de los lugares en donde advierten movimientos policiales o patrulleros, aún cuando este alejamiento les impida llegar a algún lugar al que les interesa o necesitan acceder (RC 4-6-14). Así, el temor por la policía, restringe sus experiencias cotidianas.

Otro tipo de contactos resultan en experiencias que podemos asociar con la realización de gestiones, para ellos mismos o terceros. Este tipo de contacto fue uno de los identificados al describir las razones que interfieren en la participación en los programas. Llegar tarde a las actividades, o faltar, o retirarse antes de la finalización del encuentro puede deberse a que algún familiar o amigo/a fue detenido y/o apresado, o porque el horario del programa coincidía con la posibilidad de visitar a alguien preso, o porque hubo que realizar algún trámite judicial relacionado con alguna causa propia o ajena (R39, R51, R69, R71, entrevista a VC).

El tipo más extremo de contacto, es la muerte, y en los relatos sobre las relaciones entre jóvenes y policías es un elemento cotidiano. El funcionario que creó el programa de prevención del delito Comunidades Vulnerables narró que su creación se originó a partir de la demanda generada por vecinos de un barrio popular preocupados porque “a los pibes los está matando la policía”. La muerte a manos de la policía, en estos contextos, es literal. La policía ha estado implicada en muertes de familiares de muchos de ellos (R47, entrevista YR), así como ha sido partícipe de enfrentamientos con ellos en los que casi mueren o

17 Esto se enlaza, además, con el miedo más amplio que incluye lo judicial y la dificultad que mucho tienen para comprender los procesos judiciales en los que están involucrados. 
salen muy lastimados (entrevista a NV, RC 23, RC 27-6-14); cuando la muerte no es real, cuentan que sueñan que la policía los mata (RC23). En Morón, en Avellaneda, y en San Martín, la propia muerte, o la de algún familiar o amigo, en alguna situación en la que esté contemplada la policía (enfrentamientos en el marco de delitos, casos de gatillo fácil, muertes en instituciones de encierro) puede ser parte de un pasado cercano o en el horizonte. Tal es así que en Avellaneda, y también en San Martín, los jóvenes realizaron murales alusivos a los jóvenes asesinados por la policía.

\subsection{Percepciones y valoraciones de los agentes de los programas sobre la} policía.

Los agentes (funcionarios y operadores) de estos programas de prevención social del delito y/o inclusión social quieren separarse de todo lo que tenga relación con lo policial y/o judicial penal y mostrar sus intervenciones como espacios en los que los jóvenes puedan confiar. Así parecen no reconocerse como parte del mismo Estado al que representa la policía. Desde nuestra perspectiva, esto no sólo evidencia la necesidad de entender al Estado de un modo no totalizante, sino que plantea preguntas centrales hacia el interior del funcionamiento de lo "estatal” y sus efectos en las poblaciones a las que regula. Además, la mayoría de los agentes de estos programas interpretan que la policía es fundamentalmente una institución corrupta, que se financia con actividades ilegales, que se dedica a armar causas, y que regula el tráfico ilegal de armas a las que acceden luego los jóvenes. Ahora bien, estas percepciones generalmente compartidas, adquieren matices según se trate de trabajadores de nivel territorial o de trabajadores que ocupen posiciones de coordinación de programas o de las áreas de las que estos dependen. 
“Con la policía nada”, me contestó una operadora del programa de prevención del delito en Avellaneda cuando le pregunté sobre las posibilidades de articular, habida cuenta de las conflictivas relaciones con los chicos. En la misma línea, una operadora de un Servicio Local de Morón, defendía la nula relación con la policía, y, en caso de interactuar con ella defendía una posición de enfrentamiento con la comisaría del barrio en donde estaba ubicado el servicio local. Al consultarla sobre cómo gestionar esta relación tan tensa, contestaba que no era posible hacer nada, al tiempo que acotaba, casi como último recurso que, eventualmente, funcionarios del área de articulación municipal podrían acceder a la jefatura de la comisaría con la intermediación, además, del área de seguridad municipal.

La articulación entre áreas de gestión sociales y de seguridad para el abordaje del delito juvenil es un desafío que incluye posiciones encontradas. Así, desde otra perspectiva, la profesional que había sido operadora del programa de prevención del delito durante más de 10 años en Avellaneda, pero que en el momento de la entrevista dirigía el área de Niñez (de donde dependía ahora el programa), reconocía que desde el Municipio existía una preocupación, no del todo traducida en alguna práctica concreta, alrededor del necesario vínculo entre las áreas de seguridad y las sociales. Admitía la necesidad de tal articulación pero dudaba de cuán culturalmente preparados para eso estaban los actores para trabajar en conjunto.

Por su parte, una funcionaria del área de juventud de Morón, ante la pregunta específica sobre la relación con la policía, reflexionaba que, a partir de la experiencia no del todo auspiciosa de enfrentamiento “cara a cara” con la policía, habían entendido que era complicado hacer política socio sanitaria confrontando todo el tiempo con la policía: los operadores territoriales quedaban muy expuestos, además de que tal relación resultaba peligrosa para ellos y para los jóvenes y sus familias.

Respecto de la imposibilidad de interacciones no confrontativas con la 
policía o sus mandos, tanto trabajadores de nivel territorial como de coordinación coincidían en que el principal obstáculo era la falta de capacitación en derechos de niños y jóvenes de parte de la institución. “La policía no está formada para trabajar sobre estos temas, no hay nada en su formación que les explique que ellos también pueden ser un actor que forme parte del Sistema de otro modo distinto a poner a los chicos presos”.

En suma, no obstante cierto consenso en la distancia que quiere mantenerse con la policía, aquellos trabajadores que gozan de más experiencia, incluso a nivel territorial, pero han accedido a puestos de coordinación o dirección de áreas, parecen tener una mirada más reflexiva y sobre todo menos determinante sobre el vínculo que deberían tener desde los programas con las áreas de seguridad y las policías; incluso sobre los modos de tematizar hacia adentro de los espacios de trabajo con los jóvenes la relación con la policía.

\subsection{Las formas institucionales de abordaje de la relación de los jóvenes con la policía.}

En este apartado se sistematizan las formas en las que los programas abordan la relación entre jóvenes y policía. Dentro de esta categoría de análisis la distinción más significativa resultó entre las acciones que surgían de propia motivación institucional, y las que surgían de la demanda, más o menos articulada, de los jóvenes. Respecto de las acciones que se originan como iniciativa de los programas, es posible distinguir entre: 1) las que se concentran en el trabajo con los jóvenes, dentro de las cuales se distinguen a) aquellas con carácter político, y b) aquellas con carácter instrumental y 2) las que implican una articulación interinstitucional, dentro de las cuales se distinguen las que se realizan a nivel territorial y las que suponen una interacción política a nivel de ejecución gubernamental.

Respecto del tipo de acciones concentradas en el trabajo con los jóvenes y 
surgidas como parte de motivación propia de los programas, las de carácter político se centraban en la cuestión de la violencia institucional y procuraban generar en los jóvenes una actitud crítica que la problematizara, la desnaturalizara y la situara como una consecuencia histórica, especialmente, de la última dictadura cívico militar argentina iniciada en 1976. Este era el caso de las acciones en Morón, donde según los actores -agentes de los programas y jóvenes-, la violencia institucional atraviesa la vida cotidiana en barrio. En las entrevistas, tanto los coordinadores de la sede del barrio, como los del programa a nivel municipal, coincidían en que el tratamiento de la violencia institucional era parte de un trabajo de memoria, ya que entendían que la violencia institucional era una herencia de la dictadura y, en parte, un trabajo de organización social. El programa cumpliría un doble rol, por un lado, como un espacio para analizarla, vincularla con la historia reciente, y desnaturalizarla. Y por otro, como una instancia en la cual los jóvenes pudieran volcar información de cuando sucedía en el barrio, para que desde el programa se pudiera intervenir institucionalmente. Incluso, en una de las actividades del programa de inclusión, en el marco del trabajo sobre la violencia institucional, participaron miembros de la organización Comisión por la Memoria que documenta y denuncia casos de violencia institucional (RC 23-11-11).

Así, la intención de la acción surgía de la preocupación de los operadores acerca de la naturalidad con la que los jóvenes asumían la violencia institucional. Esa naturalización representaba un obstáculo para las pretensiones que tenía el programa de que los jóvenes se comprometieran y organizaran en defensa de sus derechos humanos.

Como otra de las acciones de motivación interna y de carácter político específicas para tematizar la violencia institucional, en Morón, se realizó un análisis -que intentó ser crítico- sobre el programa de televisión Policías En 
Acción. ${ }^{18}$ En las emisiones analizadas se ilustraba un allanamiento en el barrio en cuestión en donde se implementaba el programa. Mientras los jóvenes presentes se reían de las imágenes y se entretenían reconociendo lugares y personajes del barrio, el coordinador de la actividad procuraba conducir la interpretación de lo que se mostraba hacia un cuestionamiento del accionar policial vinculado con el atropello de los derechos humanos.

También como parte de acciones de motivación interna y carácter político, un Centro Juvenil en San Martín optó por tematizar la relación con la policía a partir de la violencia institucional. Por ejemplo, al conmemorar el día por la erradicación de la violencia institucional, visitando el barrio Carcova también en la localidad de San Martín-, famoso por una masacre de gatillo fácil en 2011 en la que fueron asesinados dos adolescentes.

Ahora bien, aunque originadas en la propia iniciativa de los programas, se identificaron formas de abordaje de la conflictiva relación entre jóvenes y policías que es posible calificar de instrumentales, en la medida en que procuran brindar herramientas a los jóvenes sobre cómo actuar frente a los contactos con la policía. En el Centro Juvenil de San Martín se realizó un taller orientado a saber "qué hacer si nos detienen". Desde la coordinación del programa se proponía a los jóvenes que, estar informados era "un derecho para evitar los posibles procedimientos inadecuados”. En el programa Comunidades Vulnerables, en Avellaneda, también se optó por acciones de tipo instrumental. Se estableció un contacto con la Defensoría especializada y se visitó la sede judicial con los jóvenes para que conocieran el lugar y los actores que llevan las causas que pueden tenerlos implicados, y para que supieran que allí podían acercarse a realizar consultas. La segunda parte de la actividad consistió en que la defensora fuera al barrio en donde se hacían las actividades para describirles su función, darles algunas recomendaciones para casos en que estuvieran

18 En clave documental registra la rutina policial, especialmente aquella vinculada con intervenciones en emergencias, procedimientos judiciales, operativos de allanamientos, etc. 
implicados en algún delito o entablaran relación con la policía, y evacuar dudas generales (R47). A partir de este intercambio, la operadora del programa apeló a la relación con la Defensoría, y al hecho de que los jóvenes ya conocían el lugar, para derivarlos ante cuestiones específicas.

Otra acción, originada desde el programa y orientada instrumentalmente, fue la explicación sobre ciertas modificaciones de legislación que implicaban que unos determinados trámites se hicieran desde entonces en oficinas administrativas y no ya en oficinas judiciales o policiales. Esta acción se enmarcaba en el temor que tienen los jóvenes y sus familias hacia el sistema judicial y policial y la incidencia del mismo en que no realicen algunas gestiones necesarias para la vida cotidiana -por ejemplo, documentación de identidad- (R55).

Dentro del tipo de acciones concentradas en el trabajo con los jóvenes pero originadas por la demanda -más o menos articulada- de ellos, fue posible reunir acciones dentro de un grupo que podría llamarse de derivación o consejería. Es decir, reacciones de los programas ante acciones de los propios jóvenes o sus familias. Es habitual que tanto jóvenes como sus familias acudan a los referentes de los programas solicitando asesoramiento, ayuda, o simplemente para compartir ciertas situaciones que les sucedieron por fuera del programa en sí. Por ejemplo, citaciones judiciales que les llegaron y cuyas implicancias no entienden, o abusos policiales en detenciones. En estos casos, lo que se registró es que las acciones del programa consistían en derivar a las personas, en general, a la Defensoría (R50, R51). Sobre todo, a posteriori de que se hubiera realizado aquel contacto que mencioné en el punto anterior. También se registran intervenciones que aconsejaban a los progenitores de los jóvenes no dar intervención a la policía para solucionar problemas de índole social (como lograr que un hijo se deje internar para un tratamiento de adicciones) (R75).

Otra forma de reacción lo constituyen los consejos que los operadores 
pueden darles a los jóvenes, que procuran que éstos conozcan y pongan en práctica sus derechos. Por ejemplo, en una de las ocasiones en que uno de los jóvenes estaba procurando, con ayuda de una de las operadoras, obtener un permiso municipal para cuidar coches, el joven temía que la policía, aún con su permiso, lo detuviera. La operadora le explicaba que, con el permiso vigente y sin incurrir en acciones en contra de la ley, nadie (ni la policía) podría detenerlo u hostigarlo (R55). Estos consejos no incluían una consideración sobre la relación marcadamente desequilibrada de poder que existe entre los jóvenes, especialmente de sectores populares, y la policía, y el escaso margen que éstos tienen para colocar un discurso de derechos en sus interacciones con las fuerzas policiales.

Finalmente, como parte de acciones que surgen como motivación interna de los programas, pero que no se concentran en el trabajo de jóvenes, se encuentran las que implican algún grado de inter institucionalidad. En un extremo, podemos ubicar acciones que prácticamente constituyen rupturas más que articulaciones. En Morón se registraron situaciones en que operadoras del programa social se enfrentaron a un comisario que tenía jurisdicción en la zona, a raíz de que éste había intervenido sin razón en una operación de restitución de derechos que estaba realizando el programa.

Dentro de lo que sí pueden calificarse como articulaciones -en el sentido de mejorar las intervenciones- se ubican las colaboraciones entre programas u otras instancias territoriales. Por un lado, se despliegan articulaciones territoriales, por ejemplo, en Morón, entre el programa de inclusión juvenil con otro programa municipal cuyo objetivo es el acompañamiento territorial a familiares de presos y detenidos y el apoyo ante casos de violencia institucional. Otro tipo de articulación se constituyó, tanto en Avellaneda como en San Martín, cuando los programas participaron en foros y mesas locales de seguridad. Estos espacios son usualmente convocados y compuestos por vecinos preocupados por el delito callejero, que suelen desconfiar de los 
jóvenes y apelan a la policía para velar por el orden. Como parte de una deliberada estrategia para remover estos prejuicios hacia los jóvenes, los agentes institucionales de Avellaneda concurrieron a estos espacios para dar a conocer el trabajo realizado con los jóvenes. En 2017, en San Martín, autoridades del área del que depende el programa de acompañamiento a jóvenes en conflicto con la ley también se referían a su participación en este tipo de espacios, y hacían alusión a la necesidad de contar, en esos ámbitos “difíciles”, el objetivo y alcance de sus tareas. No obstante esas incursiones en la arena de seguridad, la mayoría de los agentes institucionales de los programas defendían que estas estrategias dependiesen de áreas sociales. Esta posición se contrastaba con la expresada por el funcionario creador del programa de prevención del delito Comunidades Vulnerables, que como se señaló antes, surgió de la Dirección de Política Criminal del entonces Ministerio de Justicia y Derechos Humanos. El funcionario defendía que la prevención social del delito juvenil estuviera ubicara en área de seguridad, dado que de no dotarse a dichas áreas de contenidos “sociales”, se llenarían de otros, posiblemente más cercanos a los represivos.

Por último, se encuentran las articulaciones inter institucionales a nivel político o de gestión, que ni se realizan a nivel territorial, ni implican trabajo con los jóvenes. Este fue el caso, por ejemplo, del programa Comunidades Vulnerables en Avellaneda, cuya operadora realizó visitas, no regulares pero tampoco excepcionales, a la sede judicial para averiguar el estado de algunas de las causas de los jóvenes bajo programa (R54). Esta acción suponía la consideración por parte de los operadores sobre lo dificultoso que es para los jóvenes la relación con el sistema judicial penal. También, desde el mismo programa se procuró entablar, muchos años después de haber empezado a trabajar en el territorio, relaciones con la Dirección de Seguridad del Municipio y reuniones con la Fiscalía departamental para, a partir de ese contacto o por intermedio de él, llegar a las jefaturas de las comisarías y dar a conocer el programa (R58). Las reuniones con la Fiscalía finalmente se realizaron, y 
aunque muy dispersas en el tiempo, incluyeron una serie de mapas del delito del barrio, en donde se incluían puntos de acceso a armas ilegales, venta de drogas, y situaciones de gatillo fácil (RC 18-3-14). Con puntos de sintonía y diferencia con la estrategia de Avellaneda, en Morón, con las informaciones que el programa recibía de parte de los jóvenes sobre allanamientos y eventuales detenciones, las operadoras podían articular con el área de Seguridad municipal para que ésta se contactara con la comisaría (y le hiciera notar que el programa estaba velando por los derechos de los jóvenes), y a la vez con el área de derechos humanos.

Ahora bien, vale aclarar que en todos los casos se trata de acciones fragmentarias, no sistemáticas y que, salvo una excepción, no figuraban en los diseños programáticos de los programas. Sólo el programa de prevención del delito “Comunidades Vulnerables” consideró en su diseño la articulación de la estrategia social con la policía, mediante un acuerdo entre la Dirección de Política Criminal de donde dependía el programa y el Ministerio del Interior del cual dependían, entonces, las fuerzas policiales. Esa doble dependencia quedó trunca a poco de empezar el Programa, con lo cual la articulación con la policía nunca se hizo. En el resto de los casos, los operadores y coordinadores de los programas reconocían que las articulaciones se establecieron una vez advertido lo infructuoso y contraproducente del choque cotidiano, o rispidez permanente con la policía que operaba en el área de influencia del programa en cuestión.

\section{DISCUSIÓN: INTERPRETACIONES SOBRE LA DISTANCIA Y SUS EFECTOS}

En el apartado anterior evidencié que la policía forma parte de la vida cotidiana de los jóvenes que están incluidos en programas de inclusión social y prevención del delito, y que en éstos la cuestión es abordada de múltiples maneras no sistemáticas. A su vez, más allá de cómo se aborde, los agentes de 
los programas tienen ideas sobre la policía y sobre qué tipo de acciones pueden realizarse para incidir en la relación que la policía tiene con los jóvenes, y con ellos mismos. Las valoraciones de los agentes inciden en las acciones que desde los programas emprenden o no emprenden. Al respecto me interesa puntualizar algunas cuestiones - a modo de líneas de indagación sobre las que habría que profundizar-, que podrían estar incidiendo en que las relaciones que desde los programas se entablan con lo policial se caractericen por la generalizada distancia, desconfianza, alteridad y hasta incluso marcada confrontación.

En primer lugar, en relación a la construcción de sentidos asociada al “cambio" 19 de paradigma de la "situación irregular" de los menores ${ }^{20}$ al de protección de derechos, con la consecuente separación de las causas asistenciales de las penales. Por un lado, es posible que dicha separación haya impactado en las dinámicas institucionales donde efectores del sistema de protección por un lado, y del sistema penal por otro, procuren no inmiscuirse en la esfera contraria para no caer en actitudes “criminalizantes” los primeros y “tutelares” los segundos. Esta preocupación está presente cuando, por ejemplo, la trabajadora de Avellaneda señalaba como “retroceso" la eventual posibilidad de que el programa de prevención del delito se inscribiera en un área de seguridad o justicia. Este rechazo a interactuar con la policía desde las áreas sociales sería más marcado en los trabajadores de base, más cercanos a la cotidianeidad juvenil, y se moderaría a medida que la jerarquía institucional es más alta: los coordinadores de programas o funcionarios de áreas directivas aceptaban, al menos, una incipiente articulación. No obstante, persisten discrepancias sobre cuál es la mejor dependencia que pueden tener los

19 Sin poder extenderme aquí, las comillas sobre el término procuran matizar la radicalidad de dicho cambio entre paradigmas, dado que se han documentado muchas más continuidades y mutaciones entre ellos que rupturas drásticas (para ampliar, cfr. Villalta y Llobet, 2011).

20 Vinculado primero con la sanción de la Convención de los derechos del niño, y luego, en Argentina, con la derogada Ley de Patronato de Menores y la sanción en 2005 de la Ley de Promoción y Protección Integral de los Derechos de niños, niñas y adolescentes. 
programas sociales orientados al tratamiento del delito juvenil, aún desde la perspectiva de funcionarios de alto rango. Con lo cual, habría que seguir indagando cuánto de estas percepciones se asocian al nivel de cercanía o distancia con la cotidianeidad juvenil que tengan los agentes institucionales (si son operadores territoriales o funcionarios), o si más vale se vincula, por ejemplo, con la procedencia disciplinar de los agentes.

En este sentido, una segunda cuestión que puede configurar el modo en que los programas sociales abordan la conflictiva relación entre jóvenes y policía es la procedencia disciplinar de los trabajadores. Quienes a diario implementan este tipo de programas en los territorios son, o bien profesionales del trabajo social y la psicología o, si acaso no son profesionales, gozan de una trayectoria o interés fuerte en las temáticas vinculadas a la pobreza, la desigualdad, la marginalidad, la vulneración de derechos. Incluso, muchos de ellos son o han sido militantes sociales, o comunitarios o políticos orientados por ideas "de izquierda" o vinculadas a alguna versión tercermundista de la religión católica. De allí que sostengan su trabajo en el marco de la defensa de los derechos humanos y encuentren en la policía al actor por excelencia en las antípodas de todo su accionar. Los programas se colocan como los que están allí, en el terreno, para proteger a los jóvenes en situación de vulnerabilidad. Tal como se detalló en las formas que abordan la relación, las intervenciones de los programas reafirman en sus intervenciones la percepción negativa de los jóvenes sobre la policía.

Aunque no parecería inadecuada esa caracterización de la policía, y las acciones de formación en derechos humanos orientadas a instruir sobre todo lo que la policía no debe hacer, así como la problematización, visibilización y denuncia de la violencia institucional son fundamentales, no deberían ser las únicas formas de abordar la cuestión. Especialmente, habría que indagar en qué medida son las más útiles para los jóvenes en su tarea cotidiana de convivir con un aparato policial que, actualmente, parece aún menos proclive que hace unos 
años a ajustarse a derecho. Siguiendo a Saín (2015), cabe preguntarse en qué medida los discursos que procuran ser más progresistas no colaboran finalmente con las políticas más reaccionarias dejando el terreno libre para que éstas se desplieguen con legitimidad. Especialmente no deberían ser las únicas acciones considerando la habitual y generalizada situación de vulnerabilidad social de los jóvenes a los que se dirigen, justamente por el ser el grupo, por excelencia, en el que se enfoca la policía para su tarea de selección de la criminalidad.

Al respecto se ubica el tercer señalamiento que interesa colocar. Por momentos, parecería que ciertas intervenciones de los programas, sobre todo aquellas que tienen a inculcar en los jóvenes los derechos que tienen ante eventuales hostigamientos policiales, o abusos, tienen un cierto desfasaje de clase, y no logran ponderar acabadamente la situación de completa inferioridad y desprotección en la que se hallan los jóvenes -especialmente varones- de sectores populares en sus encuentros con la policía. Y este “desfasaje” en el tono de los contenidos es llamativo porque los operadores de los programas, sobre todo los que se implementan territorialmente, conocen muy bien las situaciones en las que jóvenes y policías interaccionan. Parecería preciso considerar más agudamente las condiciones de posibilidad que tienen los jóvenes para negociar “in situ” y cara a cara, el acceso a sus derechos. Vale considerar, en este punto, la recomendaciones realizadas, específicamente para el trabajo con jóvenes de sectores populares en el marco de intervenciones comunitarias y/o estatales, por documentos como el Manual del Derecho a tener Derechos (Rodríguez Alzueta et al., 2009). Este tipo de materiales, además de formativos en derechos humanos, asumen las condiciones de posibilidad específicas del ejercicio de derechos que tienen los jóvenes, varones y pobres frente al accionar policial, e indican estrategias convenientes para minimizar las situaciones ilegales y de riesgo de violencia institucional que tienen como víctimas a jóvenes, sin exponerlos a mayor ensañamiento por parte de las fuerzas de seguridad. 
Finalmente, el cuarto punto es transversal a los demás, y representaría un “problema” mucho más extendido socialmente y para nada circunscrito a la especificidad de los programas sociales. Se trata de una limitada concepción de la seguridad, en general, y de la policía en particular.

En primer lugar, el concepto de seguridad y todo lo relacionado con ella, parece connotar para agentes de los programas (y posiblemente también para los jóvenes) solamente los asuntos relativos a la seguridad pública, vinculada con la prevención y represión del delito urbano contra la propiedad o contra la vida. Aún más, incluso dentro de la reducción de la seguridad a la seguridad pública, el universo de acciones posibles se acota en el control del delito, como la imposición policial del orden y no caben opciones vinculadas con la gestión de la conflictividad (Binder, 2009). Las ideas alrededor de la seguridad terminan en el universo de acción de las fuerzas de seguridad y no incluyen ni conceptos ni prácticas vinculadas a las ideas de seguridad ciudadana, pensada de una forma más integral que incluya las condiciones básicas para desarrollar una vida más o menos previsible.

En segundo lugar, y enfocando especialmente en la policía, ésta es pensada como un ente autónomo que funciona con su propia lógica independientemente de la sociedad en la cual se inscribe. Es decir, parece no considerarse que la policía, como institución, más allá de la relativa autonomía con la que puede funcionar, es el resultado -nunca del todo fijo sino más vale dinámico-, de la práctica social, y que la policía es como es en función de la relación que mantiene con la sociedad, lo que ésta le pide y la legitimidad que le otorga, aún cuando tenga una relación ambigua con ella (Saín, 2015; Galvani, 2016). Específicamente, el accionar policial es fruto de la legitimidad social que tienen ciertas prácticas, por ejemplo, el hostigamiento a ciertos sectores (jóvenes, varones, de sectores populares), en nombre de "la prevención” 
(Kessler y Dimarco, 2014). ${ }^{21}$

Conectado con el señalamiento sobre la falta de consideración de la policía como fruto de la práctica social, interesa destacar que mientras uno de los argumentos que utilizan los agentes de los programas para justificar su falta de motivación para articular acciones con la policía, es que ésta nada sabe de derechos y mucho menos de derechos de la infancia y juventud. Si bien esto resulta verosímil, igual de cierto parece ser que las áreas sociales tampoco han sido preparadas para entender a la policía como una construcción social y sus prácticas como parte de una relación social que desborda completamente a la institución. No se trata de minimizar la violencia institucional y la función represiva de la policía, ni la responsabilidad que le cabe ante esas acciones, pero sí de entender mejor sus prácticas para poder relacionarse con ella de un modo menos dañino, más respetuoso de la ley y de los derechos, y sobre todo, que considere las condiciones de posibilidad del ejercicio de derechos de los jóvenes más vulnerables.

21 Sin pretensión de abordar este punto me parece importante proponer un futuro análisis sobre cuán ajustadamente estos programas sociales destinados a jóvenes están considerando las valoraciones sobre los derechos humanos que tiene la población con la que trabajan. No resulta ninguna novedad que las ideas y prácticas más conservadoras, retrógradas y en este caso punitivas, no son patrimonio exclusivo de ninguna clase social, y que los sectores populares no están exentos de conciliar con ellas. Datos que aún tenemos en análisis dan indicios en este sentido. Uno de los operadores de un Centro Juvenil en San Martín comentaba con preocupación la validación que los jóvenes participantes habían dado a una situación de "linchamiento" (justicia por mano propia hasta producir la muerte) de un supuesto ladrón que había acontecido en las inmediaciones de su barrio. Al operador le llamó la atención esta posición de parte de los jóvenes, los cuales podrían haber estado en la posición del "linchado", ya que son a diario las víctimas del hostigamiento policial y de la estigmatización de vecinos de sus barrios. Sin necesariamente homologar la legitimación de la violencia social con la institucional, habría que considerar estos paralelismos para no dar algunas valoraciones sociales por supuestas. En ese punto, los programas también deberían considerar en su preocupación por lograr empatía e interpelación a los jóvenes más excluidos, que una postura muy confrontativa hacia las formas de accionar policial o de gestión de la seguridad no genere más repulsión que convocatoria. 


\section{REFLEXIONES FINALES}

Los programas sociales y de prevención social del delito, que combinan objetivos de cuidado y protección con otros más o menos explícitos de control y seguridad pública, orientan sus intervenciones hacia los jóvenes en base al fomento de un "proyecto de vida" centrado en la inserción educativa y laboral, y conceden particular importancia al entorno vincular de los jóvenes. Sin embargo, parecen avanzar muy tímidamente en el abordaje de una de las situaciones cotidianas que más problemas trae a los jóvenes, especialmente a los varones: una conflictiva relación con la policía. Este "silencio" respecto de la policía merece ser repensado, especialmente, en la medida en que mientras se escriben estas líneas, se vive en Argentina un recrudecimiento de formas represivas de gestionar la cuestión social en general, y debates acalorados sobre las reformas del Sistema de Responsabilidad Penal Juvenil que implicarían un ampliación de la intervención del Estado penal sobre los jóvenes. Parece menester agudizar las estrategias de protección hacia los sectores que, si en general son los más castigados, ahora se encuentran en una posición de suma vulnerabilidad.

En este sentido, el abordaje de la relación de los jóvenes con la policía que hacen los programas sociales podría pensarse en tres niveles, mutuamente informados.

El básico y más inmediato, por sus condiciones de posibilidad, debería suponer formar a los jóvenes en estrategias de relación con la policía que los protejan o minimicen sus abusos sin exponerlos a un enfrentamiento con ella, que no tienen ninguna posibilidad de ganar ni empatar. En ese sentido, las intervenciones de los programas que fomentan la hostilidad y confrontación con la policía no serían, presumiblemente, las mejores aliadas. Más vale, esta formación debería considerar una propuesta que tendiese a distanciar a los jóvenes de la policía. Por ejemplo, y es sólo una entre muchas otras posibles, 
procurando formas de resolución no violenta de conflictos familiares, barriales, interpersonales, que fueran mediables sin intervención policial ni judicial.

Este tipo de acciones, en el nivel de la implementación cotidiana, requieren, por un lado, una re discusión sobre las interpretaciones circulantes del problema que existen entre los propios operadores y que condicionan las prácticas posibles. De allí que, en un nivel medio valga preguntarse por la necesidad de espacios institucionales de reflexión, formación y capacitación a los propios funcionarios y operadores sociales sobre la policía y sus relaciones con los jóvenes, que incluya, además, las percepciones juveniles sobre dicha relación.

Finalmente, es posible suponer que estas intervenciones cotidianas se verían fortalecidas y legitimadas por articulaciones en ámbitos de mayor jerarquía institucional entre las áreas sociales y las conducciones políticas de la policía. Si bien fragmentadas, de corto alcance y esporádicas, hay experiencias de este tipo que podrían revisarse y retomarse, aún en las condiciones actuales que son, sin dudas, más hostiles que en los últimos años.

\section{REFERENCIAS BIBLIOGRÁFICAS}

Alvarado Mendoza, Arturo. (2015). Nociones de justicia, legalidad y legitimidad de las normas entre jóvenes de cinco países de América Latina. Revista Sociedade e Estado, vol. 30, N1, 75-97.

Auyero, Javier y Berti, M. Fernanda. (2013). La violencia en los márgenes. Una maestra y un sociólogo en el conurbano bonaerense. Buenos Aires: Katz Editores.

Ayos, Emilio. (2010). Delito y pobreza: espacios de intersección entre la política criminal y la política social argentina en la primera década del nuevo siglo. San Pablo: IBCCRIM.

Binder, Alberto. (2009). El control de la criminalidad en una sociedad 
democrática. Ideas para una discusión conceptual. En Kessler, Gabriel: Seguridad y ciudadanía: nuevos paradigmas, reforma policial y políticas innovadoras. Buenos Aires: Edhasa.

Bover, Tomás. (2016). Trayectorias policiales: producción de instituciones y agentes sociales en la Policía Federal Argentina. Tesis doctoral en Antropología Social. Universidad Nacional de San Martín.

Burawoy, Michael. (1998). The extended case method. Sociological Theory, vol. 16, N1, pp.4-33.

Chaves, Mariana. (2010) Jóvenes, territorios y complicidades. Una antropología de la juventud urbana. Buenos Aires: Espacio.

Cozzi, Eugenia, y Mistura, M.E. (2014). Una bronca más: policía y jóvenes de sectores populares en Rosario y Santa Fe. XI CAAS, Rosario.

Daroqui, Alcira, López, A. Laura, Cipriano García, R.F. (coord.) (2012). Sujeto de castigos. Hacia una sociología de la penalidad juvenil. Buenos Aires: Homo Sapiens.

Das, Veena \& Poole, Das. (2008). El Estado y sus márgenes. Etnografías comparadas. Cuadernos de antropología social, №27, pp.19-52.

De Marinis, Pablo (1999). Gobierno, gubernamentalidad, Foucault y los anglofoucaultianos (O: un ensayo sobre la racionalidad política del neoliberalismo). En García Selgas, F. y Ramos Torre, R. (Comp.) Globalización, riesgo, reflexividad. Madrid: CIS.

Di Leo, Pablo y Camarotti, Ana. (2013). Quiero escribir mi historia. Vidas de jóvenes en barrios populares. Buenos Aires: Biblos.

Fraser, Nancy. (1991). La lucha por las necesidades: Esbozo de una teoría crítica socialista-feminista de la cultura política del capitalismo tardío. Debate Feminista, Marzo, pp. 3-40.

Fraser, Nancy y Gordon, Linda. (1994). "Dependency" Demystified: 
Inscriptions of Power in a Keyword of the Welfare State. Social Politics, Spring.

Galvani, Mariana (2015). Cómo se construye un policía. La federal desde adentro. Buenos Aires: S. XXI.

Haney, Linne (2004). Introduction: Gender, Welfare and States of Punishment. Social Politics, 11, 3.

Haney, Linne (2010). Offending Women. Berkeley: University of California Press.

Kessler, Gabriel (2004). Sociología del delito amateur. Barcelona: Paidós.

Kessler, Gabriel (2009). El sentimiento de inseguridad. Sociología del temor al delito. Bs. As.: S. XXI.

Kessler, Gabriel y Dimarco, Sabina (2013) Jóvenes, policía y estigmatización territorial en la periferia de Buenos Aires. Espacio Abierto, vol. 22, №2, pp. 221-243.

Lerchundi, Mariana y Bonvillani, André (2015). Luchas contra la desigualdad: la marcha de la gorra como experiencia de participación de los jóvenes riocuartenses. Revista Argentina de Estudios de Juventud, vol. 9, pp. 3754.

Llobet, Valeria (coord.) (2013). Sentidos de la exclusión social. Beneficiarios, necesidades y prácticas en políticas sociales para la inclusión de niños y jóvenes en el área metropolitana bonaerense. Buenos Aires: Biblos.

Llobet, Valeria y Medan, Marina (2015): “Políticas sociales y violencias hacia las y los niños y jóvenes en Argentina”. Controversias y Concurrencias Latinoamericanas, vol. 7. pp. 127-140.

Mancini, Inés (2015). La prevención social del delito. Relaciones entre agentes estatales y jóvenes de sectores populares. Buenos Aires: Unsam edita.

Matza, David (2014). Delincuencia y deriva. Cómo y por qué algunos jóvenes 
llegan a quebrantar la ley. Buenos Aires: Siglo XXI.

Medan, Marina (2014). Distintos mensajes estatales en la regulación de la “juventud en riesgo”. Astrolabio, Nº13, pp. 313-343.

Míguez, Daniel (2010). Los pibes chorros, estigma y marginación. Buenos Aires: Capital Intelectual.

Rodríguez Alzueta, Esteban (2014): Temor y control. La gestión de la inseguridad como forma de gobierno. Buenos Aires: Futuro anterior.

Rodríguez Alzueta, Esteban, Relli, Mariana, y Apella, Gabriel (2009). Manual del Derecho a tener derechos. La Plata: Colectivo de investigación y acción jurídica (CIAJ), Galpón Sur.

Rose, Nikolas (1996). ¿La muerte de lo social? Re-configuración del territorio de gobierno. Revista argentina de sociología, V.5, Nº, enero/junio 2007. [1996]

Saín, Marcelo (2015). El leviatán azul. Política y policía en la Argentina. Buenos Aires: Siglo XXI.

Segura, Ramiro (2012). Elementos para una crítica de la noción de segregación residencial socio-económica: desigualdades, desplazamientos e interacciones en la periferia de La Plata. Quid 16 N², pp. 106-132.

Sirimarco, Mariana (Comp.). (2010). Estudiar la policía. La mirada de las ciencias sociales sobre la institución policial. Buenos Aires: Teseo.

Stake, Robert (1998). Investigación con estudio de caso. Madrid: Ediciones Morata.

Villalta, Carla y Llobet, Valeria (2011). Estado de Situación del Sistema de Protección Integral de Derechos en las Provincias de San Juan y Mendoza. PIUBAMAS - SENNAF.

Zabaleta, Alfredo, Kessler, Gabriel, Alvarado, Arturo, y Zaverucha, Jorge (2016). Una aproximación a las relaciones entre policías y jóvenes en 
América Latina. Política y gobierno, Vol. XXIII, N1, 201-229.

Recepción de artículo: julio 2017

Fecha de aceptación: noviembre 2017. 